\title{
The Electronic Structure of Threading Dislocations in GaN
}

\author{
I. Arslan and N. D. Browning \\ University of Illinois, Department of Physics, Chicago, IL 60607-7059, USA
}

Dislocations are known to exhibit a wide variety of effects that can have a significant impact on the mechanical, electrical, and optical properties of many materials. One family of materials in which the properties of dislocations are particularly intriguing is gallium nitride $(\mathrm{GaN})$ and its alloys. High performance light emitting diodes (LEDs) and lasers have been fabricated using GaN despite a density of dislocations that is high enough to destroy such properties in other III-V materials. However, while these devices function, their lifetime and efficiency are limited by the presence of these dislocations. It is not immediately clear how dislocations can have both a benign and detrimental effect, and this has led to an intensive research effort to understand their properties for both practical applications and from a basic materials sciencestandpoint.

The analysis of the electronic structure of dislocations in $\mathrm{GaN}$ is performed here by a combination of atomic resolution electron energy loss spectroscopy (EELS) in the scanning transmission electron microscope $(\mathrm{STEM})^{1}$ and multiple scattering (MS) simulations ${ }^{2}$. Figure 1 shows spectra from the bulk and a dislocation core containing an edge component to the Burger's vector. There is clearly a change in the fine structure between the bulk and the core, which arises from a change in the symmetry of the structure. However, what is perhaps more important to note is that there is no shift in onset energy observed in the spectrum from the dislocation core, suggesting that there are no localized states in the band gap and the core is electrically inađive.

While EELS provides tremendous insight into the local electronic structure of defects, it does not identify its origin. To make this determination, extensive MS simulations were performed using the FEFF8 routines ${ }^{3}$. The clusters for the simulations of the bulk and edge dislocation were constructed by taking coordinates from the Z-contrast image, while the screw and mixed dislocations were built using linear elasticity theory ${ }^{4}$. The ab-plane and c-axis components for each site were simulated separately and added in the correct ratios to take into account the experimental acquisition conditions. To check the validity of the FEFF8 codes, they were first used to simulate the bulk spectrum. Figure 2 shows that there is excellent agreement with the experimental spectrum.

The simulations for the dislocation cores shown in figure 3 are all consistent with the experimental spectra in that there are no obvious localized states in the band gap (within the $2 \mathrm{eV}$ accuracy of the simulations). Furthermore, it appears that the increase in intensity of the first peak relative to the second is greatest in the screw dislocation, where all the distortions are in the c-direction. The similar fine structure of the mixed dislocation indicates that it is affected primarily by its screw component. The edge dislocation, on the other hand, (its distortions are in the ab-plane) shows an intensity of the second peak that is higher than the first (even more than the bulk spectrum where there are no distortions). As such, it can be seen that the experimental spectrum from the core in figure 1 is actually obtained from a mixed dislocation (which was originally identified as an edge dislocation since it has an identical structure in the ab-plane). Such results indicate that the intrinsic structure of the dislocation cores appears to be electrically inactive and that any activity is a result of vacancies or impurities. This is confirmed by simulations incorporating Ga vacancies in the edge dislocation (Fig. 4), where states in the band gap are observed ${ }^{5}$. 


\section{References}

1. Xin, Y., Pennycook, S. J., Browning, N. D., Nellist, P. D., Sivananthan, S., Faurie, J. P. \& Gibart, P. (1998) Appl. Phys. Letts. 72, 2680.

2. Xin, Y., James, E. M., Arslan, I., Sivananthan, S., Browning, N. D.,Pennycook, S. J., Omnès, F., Beaumont, B., Faurie, J. P. \& Gibart, P. (2000) Appl. Phys. Letts 76, 466.

3. Ankudinov, A. L., Ravel, B., Rehr, J. \& Conradson, S. (1998)Phys. Rev. B 58, 7565.

4. Arslan, I. \& Browning, N. D. in press Phys. Rev. B

5. This research is sponsored by NSF grant number DMR 9733895.
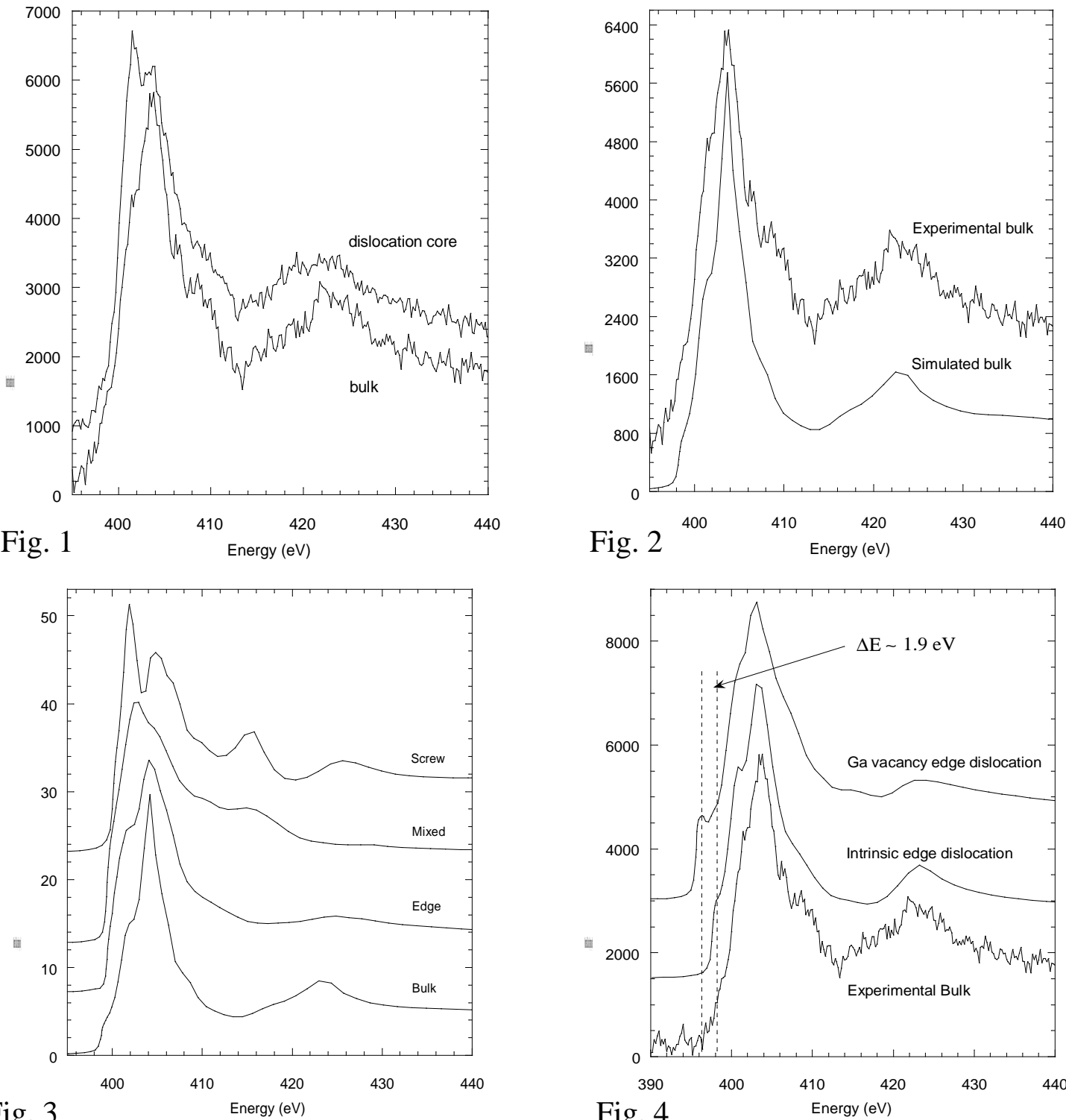

Fig. 3

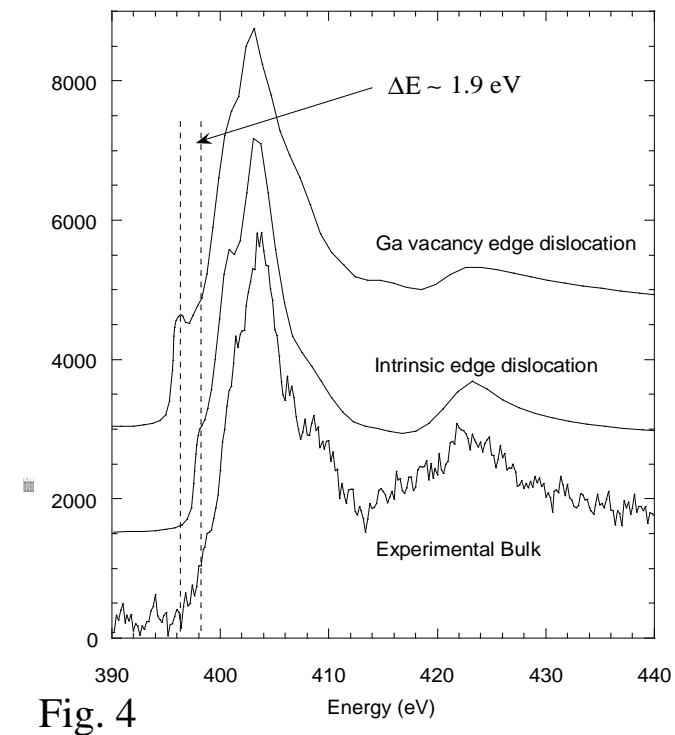

Fig. 1: N K-edge spectra from bulk GaN and a dislocation with an edge component.

Fig. 2: Comparison of experimental and simulated bulk spectra.

Fig. 3: Simulated spectra from screw, mixed and edge dislocations against bulk.

Fig. 4: Simulated spectra including Ga vacancies in the edge dislocation core. 